\title{
Collision-aware cooperative MAC protocol design for mobile ad-hoc networks
}

\author{
Y. Neeraja, V. Sumalatha \\ Department of Electronics and Communiation Engineering, JNTUA, Anantapur, AP, India
}

\begin{tabular}{|c|c|}
\hline Article Info & ABSTRACT \\
\hline Article history: & \multirow{6}{*}{$\begin{array}{l}\text { Mobile ad hoc networks are designed to maintain communication among the } \\
\text { independent nodes without a server or base station. High efficient MAC } \\
\text { protocol takes a major role to maintain collision free, bandwidth efficient } \\
\text { communication among the networked nodes. Collisions among the nodes } \\
\text { provide considerable reduction in the performance of the network. The } \\
\text { objective of the work is to provide collision aware cooperative MAC } \\
\text { protocol design by modifying the backoff to a specific range. It improves the } \\
\text { throughput and reduces delay with less collision rate. It minimizes the } \\
\text { complexity of the problem arising in memory and processing. }\end{array}$} \\
\hline Received Jun 19, 2018 & \\
\hline Revised Nov 10, 2018 & \\
\hline Accepted Jan 22, 2019 & \\
\hline Keywords: & \\
\hline MAC & \\
\hline
\end{tabular}

Copyright $(2019$ Institute of Advanced Engineering and Science. All rights reserved.

\section{Corresponding Author:}

Y. Neeraja,

Department of Electronics and Comminication Engineering,

JNTUA, Anatapuramu,

Anatapur, AP, India.

Email: neerajay30@gmail.com

\section{INTRODUCTION}

A Mobile ad-hoc network (MANET) is a collection of autonomous mobile nodes connected to each other without depending on specific fixed infrastructure [1]. Each node in the MANET can act as a router to process and transfer the packets to the destination, each node considered as a source node or destination node or intermediate node to forward the packets to the destination node. The frequent changes of nodes lead to link breakages and may fail the partial or full network communication [2]. MANETs are distributed in nature and follows multi-hop communication to transfer the data packets to the destination. Every two mobile nodes in the network can communicate each other if they are in same radio connectivity range. Less implementation cost, dynamic topological changes for connection maintenance and quick deployment are major advantages of MANETs.

\subsection{Problem Set}

The major challenges includes lack of infrastructure, mobility and dynamic nature and lack of fixed traffic concentration points, wireless communication and battery consumption, less processing power, security are the major challenges and lot of research is currently happening in these aspects. MAC layer is one of the sub layer of data link layer used to manage the collision problems in the wired and wireless networks. Generally, the MAC is used for framing, flow control, access control and physical addressing with support of other layers in the reference model. Point coordinated function (PCF) and distributed coordinated function (DCF) are two MAC layer working mechanisms. IEEE 802.11 PCF is centralized mechanism and works only with infrastructure networks and IEEE 802.11 DCF is distributed and works with ad-hoc networks and also for infrastructure networks. Channel collisions are major problems in the wireless networks including Ad-Hoc networks. 
Contention based protocols used to resolve the problems in contention; however, hidden and exposed terminal problems can arise in heterogeneous networks like mobile ad-hoc networks. The formal binary exponent backoff algorithm is unstable with long waiting queues under some assumptions. The other existing congestion control mechanisms works with large number of retransmissions.

\subsection{Background}

In ad hoc networks the communication takes place among the nodes directly with in the range or through the intermediate nodes. Various MAC issues addresses related to Quality issues, security issues, node failures, routing disturbances has been proposed by many authors. In this paper, the surveys concentrated on design issues of MAC that greatly affects the performance of MAC and provide a new scheme called cooperative semi distributed MAC routing protocol. The authors [3] a distributed MAC for wireless personal area networks, which concentrates on beacon collisions and proves that the improper slot choices, leads to frequent and repeated collisions. A self learning collision avoidance scheme [4] proposed for wireless networks, it has zero overhead in communication and considerable growth in the performance of the network.

The MAC QoS [5], [6] real time applications are discussed, such as priority reallocations and scheduling. The authors [7] discussed about the clustering schemes and multicasting routing protocol. It uses weight cost function by considering power of node, average network energy consumption and the cluster head is selected based on minimum weight value. ARAMA [8] ant routing algorithm proposed, it consists of two update mechanisms called forward packets for routing information and backward packets for updating the routing data at the individual nodes. It minimizes the congestion problem in the network and identifies the shortest possible path and reduces the end to end delay in transmissions.

Collaborative caching needs to be implemented in ad hoc networks for node mobility. Ant colony based routing scheme [9] used to identify the shortest paths among the routes using pheromone trails. The path strength is calculated based on the number of ants follows that path. Ant colony optimization proposed [10] from the foraging behavior of ant colonies and it is an optimal solution to the various NP-Hard problems. Multiple Ant colony optimizations [11] proposed for ad hoc routing. It is represented the pheromone with various colors, it is possible to allow forming better paths dynamically. An extensive study [12]-[17] carried out on IEEE 802.11 for improving the performance of the network.

The remainder of this paper is organized as follows. Sections 2 have literature review for the performance and routing aspects that motivated to the design of the new routing protocol. In section 3 , we proposed a model for new MAC protocol. Simulation results and discussions are addressed in the section 4 . Finally conclusions are drawn in section 5 .

\subsection{Objectives}

The paper objectives are following as:

a. To improve the network life time and to eliminate the possible collisions for multi hop mobile ad hoc networks.

b. To work with sensing and backoff mechanism during the cooperative behaviour.

c. To provide high efficient MAC protocol that takes a major role to maintain collision free, bandwidth efficient communication among the networked nodes.

d. To provide collision aware cooperative MAC protocol design by modifying the backoff to a specific range.

e. To improve the throughput and reduces delay with less collision rate and to minimize the complexity of the problem arising in memory and processing.

\section{METHOD}

\subsection{The Proposed Mac Protocol Design}

To improve the network life time and to eliminate the possible collisions, we present a novel CC-MAC protocol for multi hop mobile ad hoc networks. When there is a cooperative behavior exists, there is a possibility for low rate of collisions and higher network efficiency in terms of network life time, and less overhead. The proposed protocol works with several stages like sensing and backoff mechanism.

The basic operation proposed MAC scheme is following the operation of IEEE 802.11 DCF [18]. It follows the same handshaking procedure as RTS, CTS, DATA transmission, and acknowledgement (ACK) as described in the Figure 1. After getting the control packets, the NAV is to imply the time period during busy channel. 


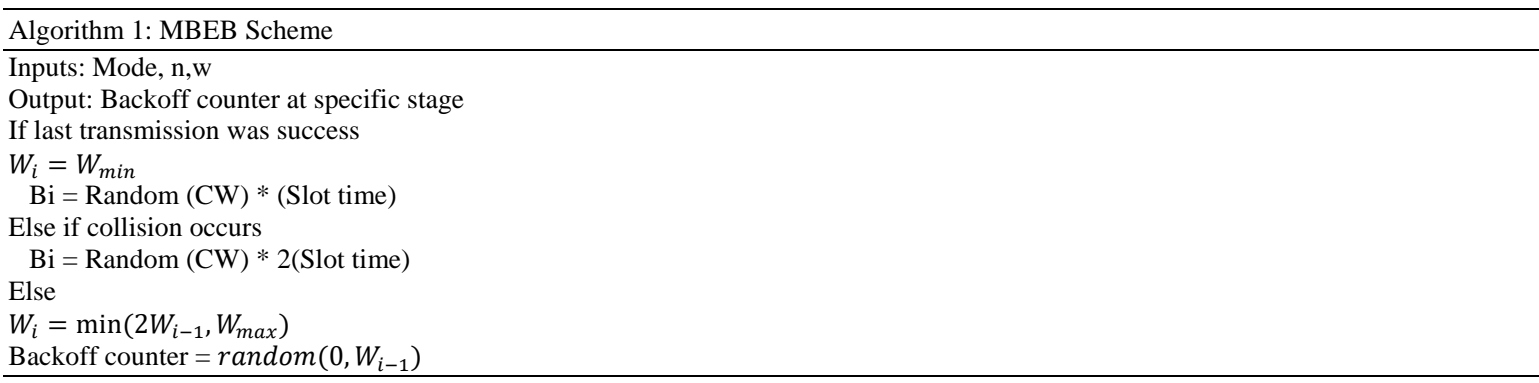

Sensing: Sensing is a process of taking approval from the destination node or from the next hop in the communication. The process takes RTS and CTS to communicate with the other nodes. DIFS.

Backoff mechanism: If channel is identified as idle for a period of (Distributed Interference space)

$B_{t}=r \times \tau=\operatorname{Random}(C W) \times \tau$

In the above equation Contention window length is calculated as $2^{r} . C W_{\min }$. The Markov backoff at sender mode is same as IEEE 802.11 but it varies at the receiver mode in relation with the Markov chain. We assumed each packet have equal priority and collisions may occur at the same probability by not considering their contention window. Random contention window from (1) determined by a random integer is from uniform distribution, that is in the range of 0 to contention window maximum and final backoff time could be the summation of $\mathrm{Bi}$ and $\mathrm{p}$.

Steady state of markov chain states that one node stays in each state can be calculated, the probability that a node transmits at the next slot is given as:

$$
\begin{aligned}
& p=\sum_{0}^{\max } p_{0}, w \\
& p=\frac{2\left(2 p_{s}-1\right)}{\left(2 p_{s}-1\right)\left(C w_{\min }\right)+\left(1-p_{s}\right) C W_{\min }\left(1-\left(2-2 p_{s}\right)^{W_{\max }}\right.}
\end{aligned}
$$

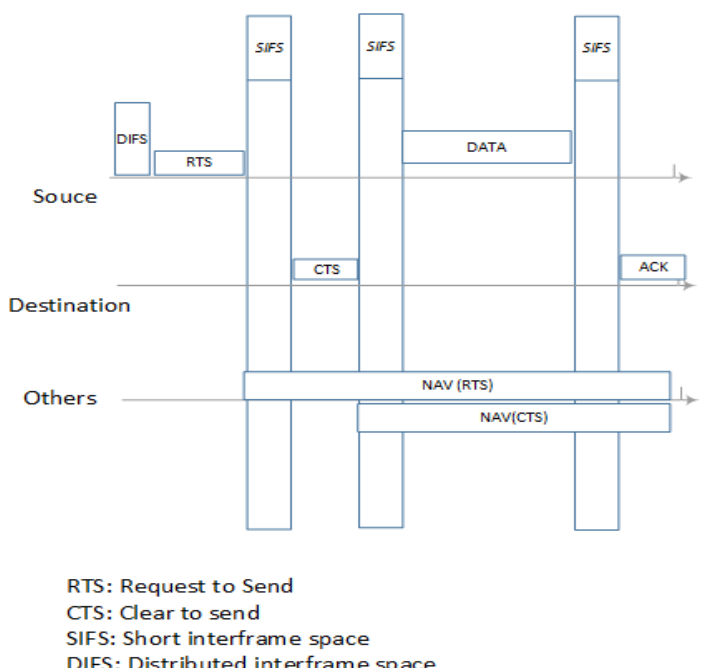

Figure 1. IEEE 802.11 DCF 


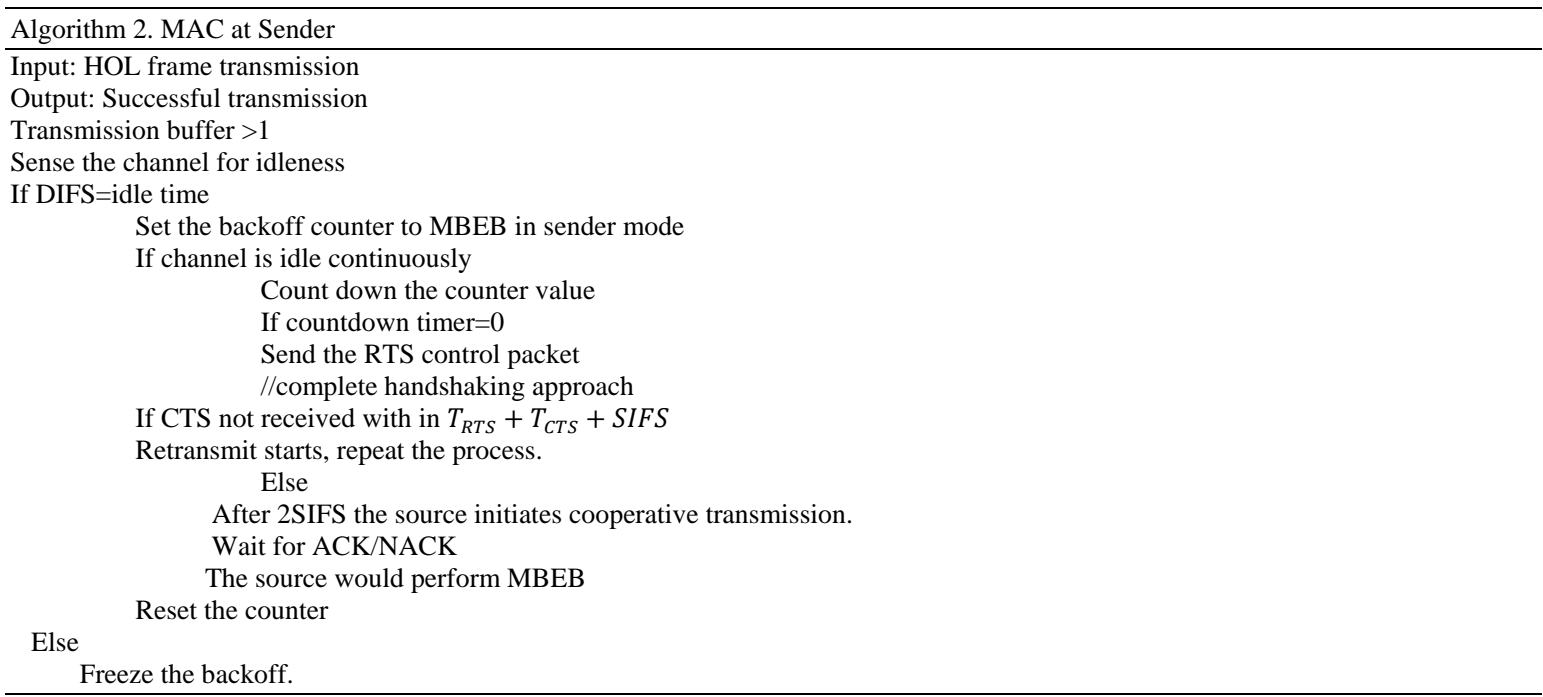

In the algorithm 2 the sender senses the channel for transmission of data, If the medium found free, it performs the handshaking process and proceed with the original data packet transfer. If idle time reaches to the DIFS period of time, it waits for MBEB backoff time and proceed with the communication. If CTS not received from the receiver, retransmission attempts should made after a specific time interval. Finally reset the counter after operation completes otherwise freeze the backoff. The algorithm 3 represents the operation at the receiver side that is based on the clear to send (CTS).

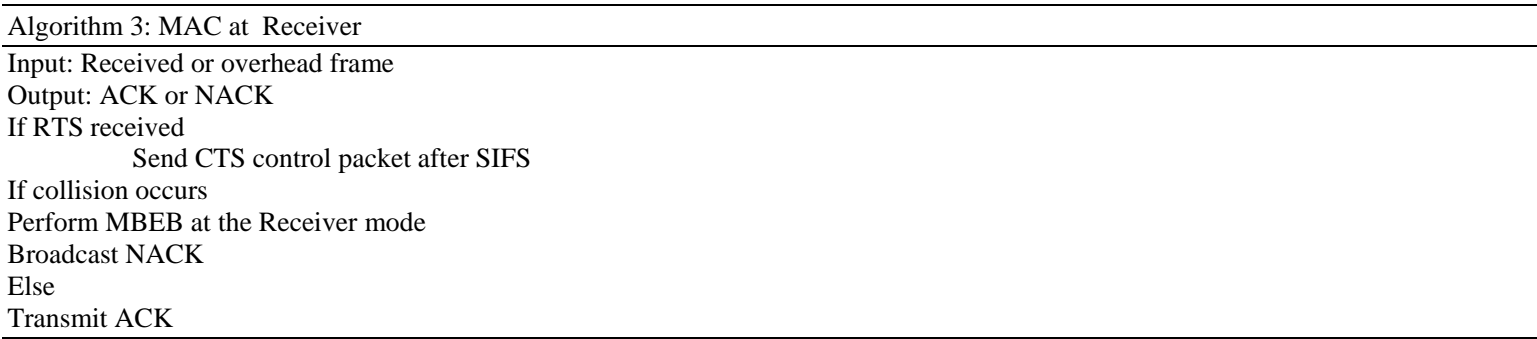

In the above process, each user has data packets and needs to transmit at different random time intervals. Before transmitting the data, each node must have to check the status of the channel for idleness. The markov chain is used for the backoff window size $1-p_{s} t o p_{s}$ with a sequence calculation of

$$
\text { Markov chain of backoff }=\left\{p_{s} / C W_{0}, \ldots, 1-p_{s} / C W_{i}, \ldots, 1-p_{s} / C W_{\max }\right\}
$$

Nodes check their individual backoff timers and generate markov chain backoff as long as distributed interference range. Collisions are possible if two or more nodes chooses the same backoff timer, it is eliminated using cooperative behavior among the nodes. Each node in the network maintains a retransmission value for the individual packet. The retransmission value is incremented every time a new retransmission occurs. If the retransmission counter exceeds 2 , it is due to congestion in the network. At this situation, the nodes identify the currently allocating capacity of the channel and adjust according to that window.

\section{RESULTS AND DISCUSSIONS}

The simulations performed using ns-2 to evaluate the performance of the proposed algorithm. The parameters described in Table 1 based on IEEE 802.11 configuration. Nodes are scattered in $100 \mathrm{~m} \times 100 \mathrm{~m}$ area with $150 \mathrm{~m}$ coverage radius. Ns-2 is an open source simulator which works effectively to perform the simulations like creating networks, changing node behaviors with custom parameters. 
Table 1. Experimental setup

\begin{tabular}{ll}
\hline Parameters & Value \\
\hline Slot Interval & 20 micro sec \\
SIFS & 10 micro sec \\
DIFS & 50 micro sec \\
Slot Interval & 20 micro sec \\
Channel Bit Rate & 2 Mbps \\
Preamble length & 144 bits \\
PCLP header & 48 bits \\
Packet length & $512 \mathrm{~KB}$ \\
CW min & 32 \\
CW max & 2048 \\
Bit Rate & CBR \\
Type & UDP \\
\hline
\end{tabular}

Collision Rate is a ratio of packets collided and total number of packets received successfully at destinations. The lower the collision rate, lesser the collisions, the simulation took 130 seconds to calculate the collision rate and are shown in Figure 2. We took the SIFS and DIFS slot intervals as 10 $\mu$ sec and $50 \mu \mathrm{sec}$ respectively.

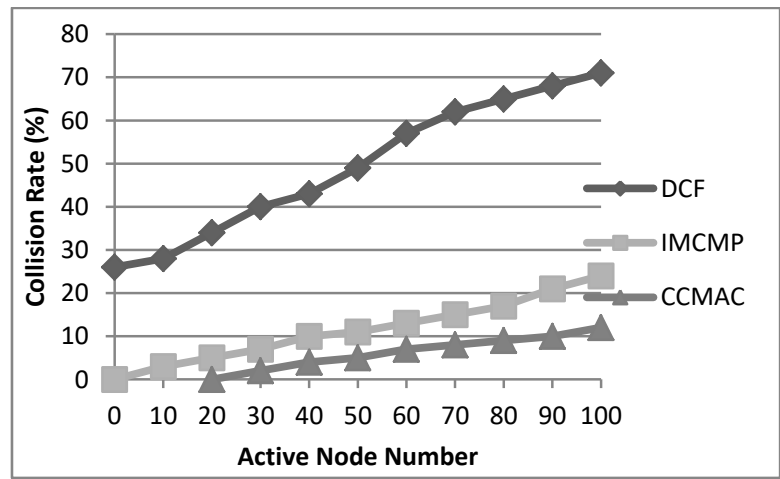

Figure 2. Collision rate vs active node number

Throughput is calculated with 150 seconds interval with respect to the channel rate. DCF performance highly affects if contending stations are more. The normalized throughput was shown in Figure 3.

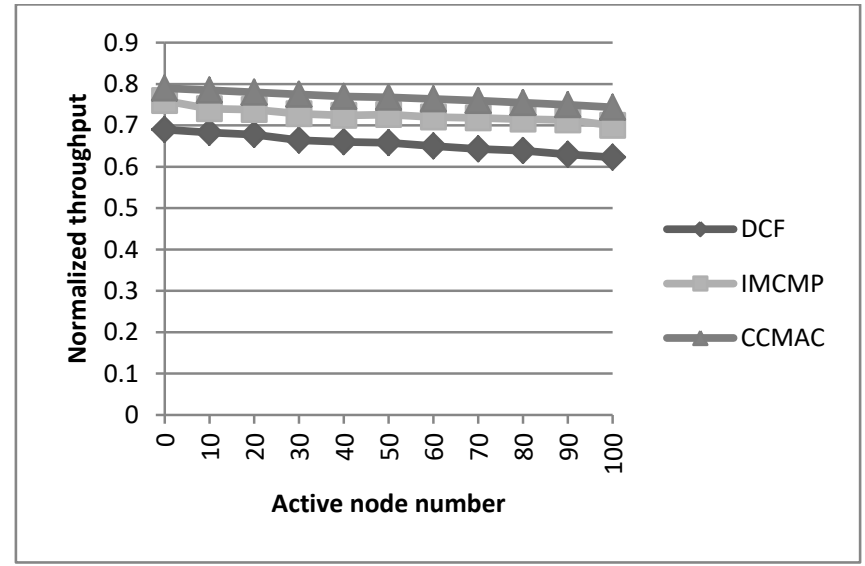

Figure 3. Normalized throughput

Figure 4 demonstrates the total offered load against control overhead comparison among the protocols such as traditional IEEE 802.11 with DUCHA, IMCMP [19]. The results obtained through the simulation 
given far better results, DUCHA is providing good performance than others. Whereas 802.11 have high control overhead [20], [21] among all compared protocols.

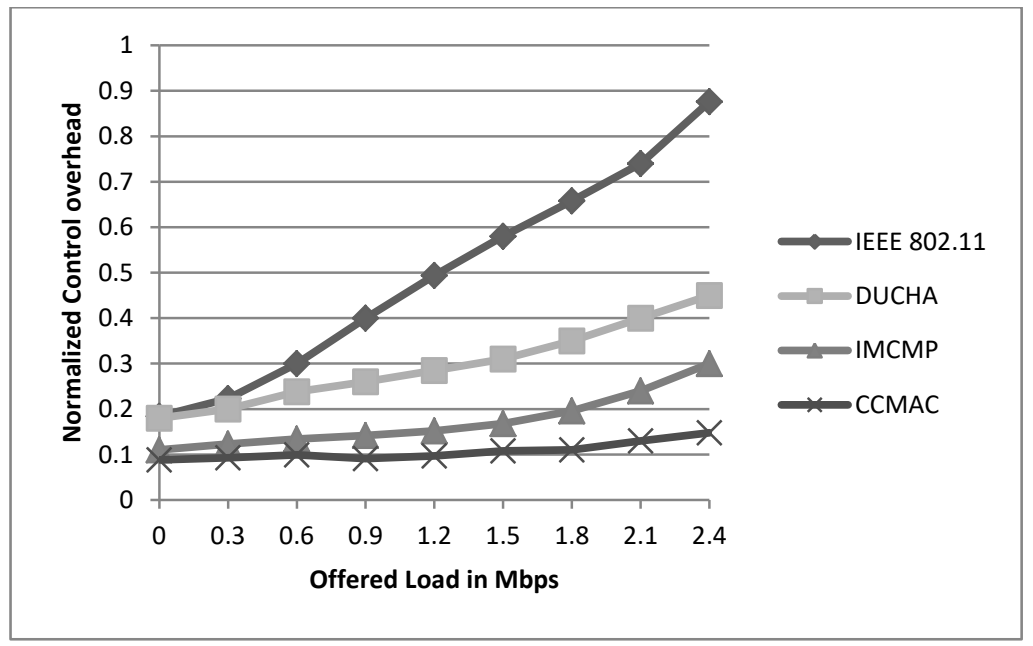

Figure 4. Total offered load vs control overhead

\section{CONCLUSION}

In this paper, we have proposed a novel collision aware scheme which follows the deterministic backoff period to minimize the collisions and improve the lifetime of the MANET nodes through cooperative retransmission behavior. The comparison results with the existing protocols given satisfactory results in the collision rate, throughput and with less overhead. It shows high impact on the improvement of the network life time by minimizing collisions using cooperative behavior.

\section{REFERENCES}

[1] Hongmei, D., Wei, L., and Agrawal, D. P., University of Cincinnati, "Routing Security in Wireless Ad Hoc Networks", IEEE Communication magazine- Telecommunication network security, Oct 2002

[2] Razzaque, M. A., Simon, D., and Paddy, N., "Cross layer self routing: a self-managed routing approach for MANETs", In proceedings of the 4th IEEE International Conference on Wireless and Mobile Computing, Networking and Communications, pp 284- 290, 2008.

[3] Vishnevsky, V. M., et al. "Study of beaconing in multihop wireless PAN with distributed control", Mobile Computing, IEEE Transactions on 7.1, pp. 113-126, 2008.

[4] Chen, C. C., et al. "Self-Learning Collision Avoidance for Wireless Networks", INFOCOM. 2006.

[5] Li, M., \& Balakrishnan, P., "Mac layer admission control and priority re-allocation for handling qos guarantees in non-cooperative wireless lans", Mobile networks and applications 10.6, pp. 947-959, 2005.

[6] Thachayani, M, \& Nakkeeran, R., "QOS Provisioning through Class-Based Route Selection for OBS Networks", International Journal of Electronics and Communication Engineering, pp. 209-219, 2010.

[7] Hwang, I. S., and Wen, H. P., "Energy efficient clustering technique for multicast routing protocol in wireless adhoc networks", IJCSNS 7.8, pp. 74-81, 2007.

[8] Hussein, O., Saadawi, T., "Ant Routing Algorithm for Mobile Ad hoc Networks (ARAMA)", International Performance Computing and Communications Conference, pp. 15-17, 2004.

[9] Joon, W., Byoung, S. C., Kyoung, T. P. L., and Ju, J., "Comparison between Heterogeneous Ant Colony Optimization Algorithm and Genetic Algorithm for Global Path Planning of Mobile Robot", In IEEE International Symposium on Industrial Electronics, pp.881-886, 2011.

[10] Dorigo, M., Birattari, M., and Stutzle, T., "Ant Colony Optimization: Artificial Ants as a Computational Intelligence Technique", IEEE Computational Intelligence Magazine, vol. 1, no.4, 28-39, 2006.

[11] Kwang, M. S., and Weng, H, S., "Ant Colony Optimization for Routing and Load-balancing: Survey and New Directions", IEEE Transactions on Systems, Man and Cybernetics, vol.33, no. 5, pp.560 - 572, 2003.

[12] Tinnirello, I., and Bianchi, G., "Rethinking the IEEE 802.11e EDCA performance modeling methodology", IEEE/ACM Trans. Netw.,vol. 18, no. 2, pp. 540-553, Apr. 2010.

[13] Cali, F., Conti, M., Gregori, E., "IEEE 802.11 Protocol: Design and performance evaluation of an adaptive backoffmecha-nism”, IEEE J. Sel. Areas Commun., vol. 18, no. 9, pp. 1774-1786, Sep. 2000. 
[14] Krishna, P. V., Misra, S., Obaidat, M. S., Saritha, V., "Virtual backoff algorithm: An enhancement to 802.11 medium-access control to improve the performance of wireless networks," IEEE Trans. Veh. Technol., vol. 59, no. 3, pp. 1068-1075, 2010.

[15] Toledo, A., Vercauteren, T., Wang, X., "Adaptive optimization of IEEE 802.11 DCF based on Bayesian estimation of the number of competing terminals", IEEE Trans. Mobile Comput., vol. 5, no. 9,pp. 1283-1296, Sep. 2006.

[16] Deng, D., Ke, C., Chen, H., \& Huang, Y., "Contention window optimization for IEEE 802.11 DCF access control”, IEEE Trans.WirelessCommun., vol. 7, no. 12, pp. 5129-5135, Dec. 2008.

[17] Trivedi, R., "Coverage and Connectivity Issue in Wireless Sensor Networks", Indonesian Journal of Electrical Engineering and Informatics (IJEEI), vol. 1, no. 2, pp. 49-52, 2013.

[18] Wireless LAN Medium Access Control (MAC) and Physical Layer (PHY) Spec, IEEE 802.11 Standard, 1999.

[19] Siyu, D., Chengdu, C., "Research of improved Multi-channel MAC Protocol in MANET", IEEE, 2017.

[20] Maheswari, R., \& Kiran Kumar, T.V.U., "International Journal of Electronics and Communication Engineering", Volume 6, Number 3, pp. 199-204, 2013.

[21] Velmurugan, V., and Martin Leo Manickam, J., "Characteristic Analysis of Efficient and Reliable Broadcast in Vehicular Networks", International Journal of Electronics and Communication Engineering. vol. 6, no. 3, pp. 205210, 2013. 